\title{
Hybrid Adaptive Control of the Boost Converter
}

\author{
Sabrina Hadjeras, Carolina Albea and Germain Garcia
}

\begin{abstract}
This work proposes a control law for the boost converter with unknown constant resistive load. It focuses on Hybrid Dynamical System (HDS) theory, which considers the voltage and current signals as continuous-time variables and the switching signal as discrete-time variable. In several applications the voltage has to be constant. To ensure that the voltage value is robust with respect to any reference, an adaptive scheme is proposed. This adaptation is accomplished using a state observer and assuming that all states are accessible. Then, the full system stability can be established by using a singular perturbation analysis. The hybrid adaptive controller is tested in simulations.
\end{abstract}

Index Terms - Hybrid dynamical system, adaptive control, boost converter, singular perturbation analysis.

\section{INTRODUCTION}

Boost converter systems are very common in power electronics. They allow to generate an output voltage larger than its input one. The control problem of this converter were largely studied by the electronic and automatic communities, by using averaged models, whose controlled signal is generally obtained by a Pulse Width Modulation (PWM) with fixed frequency [1],[2], generating a limitation in its performance.

Nowadays, there is another attempt to control this kind of converter taking into account the real nature of the system signals, that means, the fact that the voltage and current signals are continuous-time and the switching signals are discrete-time. This consideration captures the switching dynamics and achieves a new generation of converters that can operate in varying frequency. This is the case of the sliding-mode control [3] and hybrid dynamical control [4], [5], [6] for the boost converter.

Some adaptive controls for the boost converter were proposed by using also averaged models, as in [7], where a Simple Adaptive Control (SAC) was designed by using a proportional and integral controller. In [8], the authors used Lyapunov design techniques. In [9],[10] adaptingbackstepping was used to update all the parameters. On the other hand, in [11], an adaptive sliding mode control was designed, however, and to the authors best

S. Hadjeras and C. Albea ares with CNRS, LAAS, 7 avenue du colonel Roche, F-31400 Toulouse, France and Univ. de Toulouse, UPS, LAAS, F-31400 Toulouse France shadjera, calbea@laas.fr

G. Garcia is with both CNRS, LAAS, 7 avenue du colonel Roche, F-31400 Toulouse, France and Univ. de Toulouse, INSA, LAAS, F31400 Toulouse France garcia@laas.fr knowledge, to regard that the load is unknown and constant is not done in the context of hybrid dynamical paradigm.

In this work, we extend the hybrid dynamical controller proposed in [4], designing a hybrid adaptive control for the boost converter with unknown resistive load. This controller must regulate the voltage in a desired value. This adaptation is fed by a state observer designed under the consideration that the states are measurable. Then, uniformly locally asymptotically stability is ensured based on scale-time separation and using a singular perturbation analysis

The paper is organized as follow: In Section II, a model is defined under some assumptions and the problem is stated. Section III is devoted to design an adaptive law to adapt the resistive load. Section IV proposes a hybrid dynamical control that achieves the objectives of our problem. Section V deals with the proof of our main result. Some simulations are performed in Section VI. And Section V draws the conclusion and future works.

Notation: Through out the paper $\mathbb{R}$ denotes the set of real numbers, $\mathbb{R}^{n}$ the n-dimensional euclidean space and $\mathbb{R}^{n \times m}$ the set of all real $m \times n$ matrices. The set $\mathcal{S}^{n}$ denotes the set of symmetric positive definite matrices of matrices $\mathbb{R}^{n \times n} \cdot \mathfrak{R e}(z)$ is the real part of a complex number $z$. The symbol $\not \equiv$ means not identically equal.

\section{BOOST CONVERTER}

A boost converter generates an DC output voltage larger than its DC input. This converter is fed by a constant voltage source, $V_{i n}$, and composed of a load filter, $L, C_{0}$, a purely and resistive load, $R_{0}$, and a parasite resistance, $R_{L S}$, that encompasses switching energy dissipation and the inductance (see Fig. 1). The system differential equations are

$$
\frac{d}{d t}\left[\begin{array}{l}
i_{L}(t) \\
v_{C}(t)
\end{array}\right]=\left[\begin{array}{cc}
-\frac{R_{L S}}{L} & -\frac{1}{L_{L}} u \\
\frac{1}{C_{0}} u & -\frac{1}{R_{0} C_{0}}
\end{array}\right]\left[\begin{array}{l}
i_{L}(t) \\
v_{C}(t)
\end{array}\right]+\left[\begin{array}{c}
\frac{V_{i n}}{L} \\
0
\end{array}\right],
$$

where, $i_{L}$ is the inductance current, $v_{C}$ is the capacitor voltage, which are the two continuous-time state variables. On the other side, $u=\{0,1\}$ is the control input which is a discrete variable that takes the values $u=0$ and $u=1$, that correspond to the case when the switch is $\mathrm{OFF}$ and $\mathrm{ON}$, respectively.

Assumption 1: Let consider

- the converter current is continuous and all the components are ideal,

- the current and voltage are accessible, 
- the load $R_{0}$ is unknown constant or/and slowly variable in the interval $\left[R_{0}^{m}, R_{0}^{M}\right]$.

- $x_{2}=0$ corresponds to the starting operation mode, and any starting strategy is used, to bring our system to $x_{2} \neq 0$.

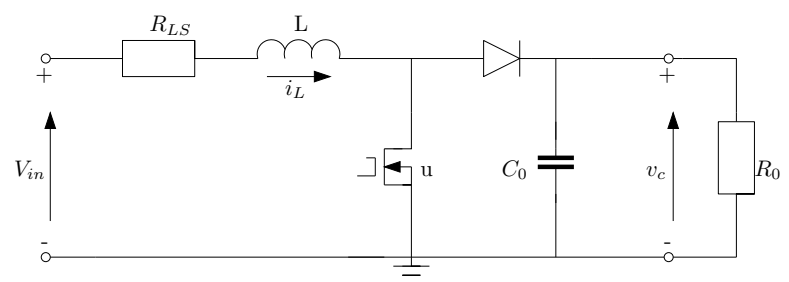

Fig. 1. Boost converter .

Defining $\beta:=\frac{1}{R_{0}} \in\left[\beta^{m}, \beta^{M}\right]$, let rewrite (1) as follows

$$
\begin{aligned}
& \dot{x}=A_{u}(\beta) x+a \\
& y=C x
\end{aligned}
$$

where $x=\left[\begin{array}{ll}x_{1} & x_{2}\end{array}\right]^{T}=\left[\begin{array}{ll}i_{L} & v_{C}\end{array}\right]^{T}$ and $C$ is the identity matrix with suited dimension. We can easily deduce the definition of $A_{u}(\beta)$ and $a$ from (1).

This paper focus on the design of a switching signal $u$, that ensures the suitable convergence properties of both: the voltage $x_{2}$ to a desired value, $x_{2_{e}}$, for the switching system with arbitrary switching, and the estimation of the load $\hat{\beta}$ to its real value, $\beta$.

Assumption 2: There exists $\lambda_{e}=\left[\lambda_{e, 1}, \lambda_{e, 2}\right]$ satisfying $\lambda_{e, 1}+\lambda_{e, 2}=1$, such that the following convex combination holds:

$$
\sum_{i=1}^{2} \lambda_{e, i}\left(A_{i}(\beta) x_{e}+a\right)=0 .
$$

This assumption is necessary and sufficient to guarantee the existence of a switching signal that ensures forward invariance of the point $x_{e}$.

Assumption 3: Let consider next polytope for parameter $\beta$ :

$$
\Omega:=\sum_{m=1}^{2} \lambda_{\beta, m} \beta_{m}, \text { for all } 0 \leq \lambda_{\beta, m} \leq 1, \sum_{m=1}^{2} \lambda_{\beta, m}=1,
$$

where the vertices of the polytope are given by $\beta_{m} \in$ $\left\{\beta^{m}, \beta^{M}\right\}$.

Now, from [4], we assume next property of the boost converter.

Property 1: Consider Assumption 3, then given matrices $A_{i}(\beta), i \in\{1,2\}$ in (2) with $\beta \in\left[\beta^{m}, \beta^{M}\right]$, there exists matrices $P, Q \in \mathcal{S}^{2}$ satisfying

$$
A_{i}^{T}\left(\beta_{m}\right) P+P A_{i}\left(\beta_{m}\right)+2 Q<0
$$

for all $i, m \in\{1,2\}$.

Note that Property 1 assumes that all matrices $A_{i}^{T}\left(\beta_{m}\right)$ are Hurwitz.

Inspired by the work in [12], this paper extends the work presented in [4], for model (2) where parameter $\beta$, can suffer variations.
Problem 1: The goal is to include an adaptive controller in the hybrid dynamical scheme that considers the continuous-time dynamics, $x_{1}, x_{2}, \hat{\beta}$, and the discretetime dynamic, $u$, estimating $\beta$ in continuous-time, at the same time that $x_{2}$ is regulated in a reference value (see Fig. 2).

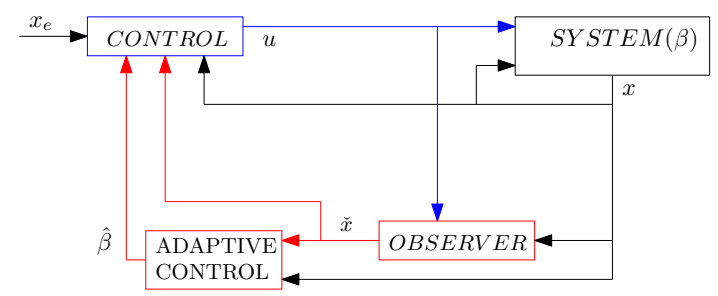

Fig. 2. Hybrid adaptive control scheme.

\section{AdAptation LAW}

The proposed adaptation law is composed of a state observer for the voltage $x_{2}$, and an adaptation law for parameter $\beta$, with the following structure

$$
\begin{aligned}
\dot{\hat{x}}_{2} & =\frac{1}{C_{0}}\left(u x_{1}-\hat{\beta} x_{2}\right)+\alpha\left(x_{2}-\hat{x}_{2}\right) \\
\dot{\hat{\beta}} & =g\left(x_{2}, \hat{x}_{2}\right),
\end{aligned}
$$

where $g\left(x_{2}, \hat{x}_{2}\right)$ is the adaptation law to be designed, $\hat{x}_{2}$ is the estimated state of $x_{2}, \hat{\beta}$ is the estimated value of $\beta$ and $\alpha$ is a positive constant parameter, which represents the convergence speed of the observer.

In order to achieve a mathematical expression for $g\left(x_{2}, \hat{x}_{2}\right)$, let define the following error variables, considering $\dot{\beta}=0$

$$
\breve{x}_{2}:=x_{2}-\hat{x}_{2}, \quad \tilde{\beta}:=\beta-\hat{\beta}, \quad \dot{\tilde{\beta}}=-\dot{\hat{\beta}}
$$

Next, from (2) and (4), we derive the error equation of $x_{2}$

$$
\dot{\breve{x}}_{2}=-\frac{\tilde{\beta}}{C_{0}} x_{2}-\alpha \breve{x}_{2} .
$$

Now, let introduce the next candidate Lyapunov function

$$
W=\frac{1}{2}\left(\breve{x}_{2}^{2}+\frac{\tilde{\beta}^{2}}{\gamma}\right),
$$

where $\gamma$ is constant and positive and

$$
\dot{W}=-\alpha \breve{x}_{2}^{2}-\frac{x_{2} \breve{x}_{2}}{C_{0}} \tilde{\beta}+\frac{\tilde{\beta} \dot{\tilde{\beta}}}{\gamma}=-\alpha \breve{x}_{2}^{2}+\tilde{\beta}\left(-\frac{x_{2} \breve{x}_{2}}{C_{0}}+\frac{\dot{\tilde{\beta}}}{\gamma}\right) \text {. }
$$

The adaptation law is now defined by canceling the terms in parentheses, i.e.

$$
\dot{\tilde{\beta}}=\frac{\gamma x_{2} \breve{x}_{2}}{C_{0}} .
$$

Remark 1: Note that, $\gamma$ defines the adaptation speed, and consequently, if $\gamma$ is larger, then the adaptation speed comes larger. 
Stability properties of (7), (9) are stated in the following lemma:

Lemma 1: Consider the system (2), and assume that its solutions are bounded. The extended observer (7)-(9) has the following properties:

i) The estimated states $\hat{x}_{2}, \hat{\beta}$ are bounded.

ii) $\lim _{t \rightarrow \infty} \hat{x}_{2}(t)=x_{2}(t)$.

iii) $\lim _{t \rightarrow \infty} \hat{\beta}(t)=\beta$, if and only if $x_{2}(t) \neq 0, \forall t \geq 0$.

Proof: The observer and the adaptive law error equations are fully defined from (7) and (9), and stability properties of these equations follow from the Lyapunov function $W$ defined above. Note that with the choice (9)

$$
\dot{W}=-\alpha \breve{x}_{2}^{2}
$$

and from standard Lyapunov arguments, it follows that the error variable $\breve{x}_{2}$ and $\tilde{\beta}$ are bounded. In addition by LaSalle invariant principle and from Assumption (1), that specifies that $x_{2} \neq 0$, we easily conclude that $\breve{x}_{2} \rightarrow 0$, which implies from (9) that $\dot{\tilde{\beta}} \rightarrow 0$. Likewise from (7), and concluding from $\breve{x}_{2} \rightarrow 0$ that $\dot{\breve{x}}_{2} \rightarrow 0$, we get $\tilde{\beta} \rightarrow 0$.

\section{HYBRID MODEL AND PROPOSED CONTROL LAW}

In this section we design an hybrid dynamical system, following the paradigm given in [13], wherein continuoustime behavior encompasses the evolution of $x, \hat{x}_{2}$ and $\hat{\beta}$ and, the discrete-time behavior captures the jump of the switch boost converter signal, $u$, and the jump of a discrete signal, $q \in\{1,2\}$, which detects if the parameter $\beta$ needs to be adapted. $q=1$ allows the $\beta$ adaptation and $q=2$ interdicts this possibility.

We characterize the overall dynamics

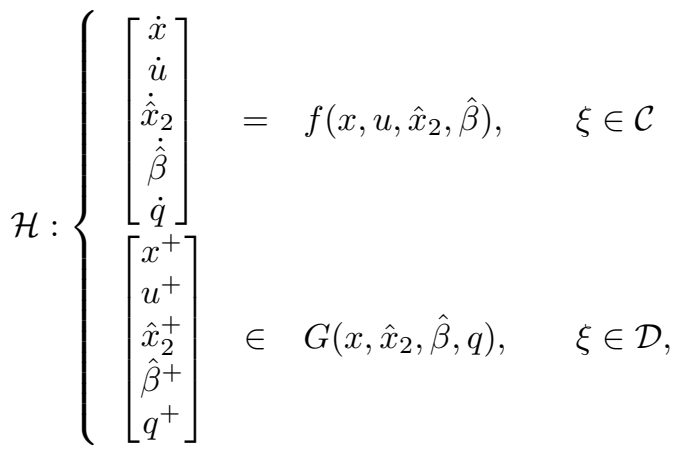

where $\xi=\left[\begin{array}{lllll}x & u & \hat{x}_{2} & \hat{\beta} & q\end{array}\right]$ and $G$ is a (set-valued) map representing the switching logic:

$$
\begin{aligned}
f\left(x, u, \hat{x}_{2}, \hat{\beta}\right):=\left[\begin{array}{c}
A_{u}(\beta) x+a \\
0 \\
\frac{1}{C_{0}}\left(u x_{1}-\hat{\beta} x_{2}\right)+\alpha\left(x_{2}-\hat{x}_{2}\right) \\
-\frac{\gamma x_{2}\left(x_{2}-\hat{x}_{2}\right)}{C_{0}} \\
0 \\
x
\end{array}\right] \\
G\left(x, \hat{x}_{2}, \hat{\beta}, q\right):=\left[\begin{array}{c}
\underset{i \in \mathbb{K}}{\operatorname{argmin}} \tilde{x}^{T} P\left(A_{i}(\hat{\beta}) x+a\right) \\
\hat{x}_{2} \\
\hat{\beta} \\
3-q
\end{array}\right]
\end{aligned}
$$

and where $\tilde{x}=\left[\begin{array}{ll}\tilde{x}_{1} & \tilde{x}_{2}\end{array}\right]$ is defined from the desired values $x_{e}=\left[\begin{array}{ll}i_{e} & v_{e}\end{array}\right]^{T}=\left[\begin{array}{ll}x_{e 1} & x_{e 2}\end{array}\right]^{T}$ as follows

$$
\left\{\begin{array}{l}
\tilde{x}_{1}=x_{1}-x_{e 1} \\
\tilde{x}_{2}=\left(x_{2}-x_{e 2}\right)+\left(x_{2}-\hat{x}_{2}\right) .
\end{array}\right.
$$

Inspired in [4], we select the so-called flow and jump sets

$$
\begin{aligned}
& \mathcal{C}_{1}:=\left\{\left(x, \hat{x}_{2}, q\right):\{q=1\} \text { and }\left|x_{2}-\hat{x}_{2}\right| \geq \varepsilon\right\} \\
& \mathcal{D}_{1}:=\left\{\left(x, \hat{x}_{2}, q\right):\{q=1\} \text { and }\left|x_{2}-\hat{x}_{2}\right| \leq \varepsilon\right\}, \\
& \mathcal{C}_{2}:=\left\{\xi:\{q=2\} \text { and }\left|x_{2}-\hat{x}_{2}\right| \leq \varepsilon\right. \text { and } \\
&\left.\tilde{x}^{T} P\left(A_{u}(\hat{\beta}) x+a\right) \leq-\eta \tilde{x}^{T} Q_{u} \tilde{x}\right\}, \\
& \mathcal{D}_{2}:=\left\{\xi:\{q=2\} \text { and }\left|x_{2}-\hat{x}_{2}\right| \geq \varepsilon\right. \text { or } \\
&\left.\tilde{x}^{T} P\left(A_{u}(\hat{\beta}) x+a\right) \geq-\eta \tilde{x}^{T} Q_{u} \tilde{x}\right\},
\end{aligned}
$$

where $\mathcal{D}:=\mathcal{D}_{1} \cup \mathcal{D}_{2}$ and $\mathcal{C}:=\mathcal{C}_{1} \cap \mathcal{C}_{2}, \eta \in(0,1)$ and $\varepsilon>0$ is small enough.

Proposition 1: The hybrid dynamical system (10)(16) satisfies the basic hybrid conditions [13, Assumption 6.5], then it is well-posed.

Proof: The hybrid dynamical system (10)-(16) satisfies the basic hybrid conditions because

- sets $\mathcal{C}$ and $\mathcal{D}$ are closed.

- $f$ is a continuous function, thus it trivially is outer semicontinuous and convex. Moreover, is locally bounded.

- $G$ is closed, then it also is outer semicontiunuous [13, Lemma 5.1] and, it is locally bounded.

Finally, following [13, Theorem 6.30], we conclude that the hybrid dynamical system is well-posed.

Now, we invokes the lemma presented in [4].

Lemma 2: Consider matrices $P, Q \in \mathcal{S}^{2}$ satisfying Property 1 , a point $x_{e} \in \mathbb{R}^{2}$ satisfying Assumption 2 . Then for each $x \in \mathbb{R}^{2}$,

$$
\min _{i \in \mathbb{K}} \tilde{x}^{T} P\left(A_{i}(\beta) x+a\right) \leq \min _{i \in \mathbb{K}}-\tilde{x}^{T} Q_{i} \tilde{x}
$$

The proof is given in [4].

Remark 2: We note here that, if $\tilde{x} \neq 0$

$$
-\tilde{x}^{T} Q \tilde{x}<-\eta \tilde{x}^{T} Q \tilde{x} .
$$

Note, that if $\eta \rightarrow 0$, we get to reduce the switching frequency and on the contrary if $\eta \rightarrow 1$, we increase the switching frequency. On the other hand, if $\tilde{x}=0$, we get arbitrary fast switches, that can be reduced using the space regularization propose by the authors in [14]. We comment here the behavior of any solution to hybrid system (10)-(16). Note that if any solution is in $\mathcal{C}_{1}$ or $\mathcal{D}_{1}$, that means $q=1$, the adaptation of the parameter $\beta$ is possible and, the contrary case is, if any solution is in $\mathcal{C}_{2}$ or $\mathcal{D}_{2}$, that means $q=2$. First, consider that any solution is in $\mathcal{C}_{1}$, then the observer error $\breve{x}_{2}$ is larger than any small and positive $\varepsilon$ and the parameter $\beta$ is adapting. When the adaptation error of $\beta$ is arbitrary small, that means $\breve{x}_{2} \leq \varepsilon$, then, the solution will be in $\mathcal{D}_{1}$ and the solution jumps. After the jump, we have $q=2$ and the solution is in $\mathcal{C}_{2}$ until that one of theses cases happens: 
- $\left|x_{2}-\hat{x}_{2}\right| \geq \varepsilon$ : then solution is in $\mathcal{D}_{2}$ and jumps to $\mathcal{C}_{1}$, flowing here until $\breve{x}_{2} \leq \varepsilon$.

- $\tilde{x}^{T} P\left(A_{u}(\hat{\beta}) x+a\right) \geq-\eta \tilde{x}^{T} Q_{u} \tilde{x}$ and $\left|x_{2}-\hat{x}_{2}\right| \leq \varepsilon$ : then solution is in $\mathcal{D}_{2}$, it jumps to $\mathcal{D}_{1}$ and following the result of Lemma 2 the solution jumps again to $\mathcal{C}_{2}$, flowing here for a time (as proven bellow).

Remark 3: Note that when $\left|x_{2}-\hat{x}_{2}\right| \geq \varepsilon$, any solution to $\mathcal{H}$ flows in $\mathcal{C}_{1}$ with $u$ constant. Then the state $x$ will flow according (1) to

$$
\begin{aligned}
i_{L, c} & =\frac{V_{i n}}{R_{L S}+u^{2} R_{0}} \\
v_{C, c} & =u R_{0} i_{L, c} .
\end{aligned}
$$

Note that if $u=0, x_{2}$ converges to $v_{C, c}=0$, however from (1), we have $x_{2}(t)=x_{2}(0) e^{-\frac{1}{R_{0} C_{0}} t}$, then $x_{2} \not \equiv 0$, $\forall t \geq 0$.

Now, following the system hybrid theory, we will establish stability properties of the given compact attractor

$$
\mathcal{A}:=\left\{\xi: x=x_{e}, u \in\{0,1\}, \hat{x}_{2}=x_{2}, \hat{\beta}=\beta, q \in\{1,2\}\right\} .
$$

The following theorem is the main result of our paper. and its proof is given in Section V.

Theorem 1: Consider Assumption 1,2,3 and matrices $P, Q \in \mathcal{S}^{2}$ satisfying Property 1 and $\gamma>0$. Then attractor (19) is uniformly locally asymptotically stable (ULAS) for hybrid system (10)-(16).

While the proof of this Theorem is given in the next Section, let comment here the choice of matrices $P$ and $Q$. These matrices are selected following some optimization criteria for our hybrid system. Specifically, we use [4, Theorem 2], where some performance level is guaranteed, then the following bound holds along any solution of the hybrid system (10)-(16)

$$
J \leq \eta^{-1} \tilde{x}^{T} P \tilde{x}
$$

defined in a compact hybrid time domain [13, Definition 2.3]. However, the resulting solutions will be sub-optimal, because they are characterized by a Fillipov solution.

On the other hand, we find that the suboptimal-level corresponds to an arbitrary high frequency switches, what can increase the dissipated energy. Therefore, we need to find a trade-off between any performance level and switching frequency.

\section{Proof of Theorem 1}

This section is dedicated to the proof of our main result. For this, we apply singular perturbation analysis due to fast actuators in hybrid control given in [?] assuming that there are slow time-continuous variables, $\xi_{1}:=(x, u, q)$, and fast time-continuous variables, $\xi_{2}:=$ $\left(\hat{x}_{2}, \hat{\beta}\right)$, then we apply singular perturbation analysis due to fast actuators in hybrid control given in [?] to establish the stability properties. For this, we will rewrite the complete system in singular perturbation form.

\section{A. Singular perturbed form}

In order to put the system above in the standard singular perturbation form, let define $\nu:=\frac{1}{\alpha}, \bar{\alpha}:=\frac{1}{\alpha C_{0}}$ and $\bar{\gamma}:=\frac{\gamma}{\alpha C_{0}}$.

With these considerations, let rewrite the hybrid scheme (10)-(11) as follows:

$\mathcal{H}_{p}:\left\{\begin{array}{l}{\left[\begin{array}{c}\dot{x} \\ \dot{u} \\ \nu \dot{\hat{x}}_{2} \\ \nu \hat{\hat{\beta}} \\ \dot{q}\end{array}\right]:=\left[\begin{array}{c}A_{u}(\beta) x+a \\ 0 \\ \bar{\alpha}\left(u x_{1}-\hat{\beta} x_{2}\right)+\left(x_{2}-\hat{x}_{2}\right) \\ -\bar{\gamma} x_{2}\left(x_{2}-\hat{x}_{2}\right) \\ 0\end{array}\right] \quad \xi \in \mathcal{C}} \\ x \\ {\left[\begin{array}{c}x^{+} \\ u^{+} \\ \hat{x}_{2}^{+} \\ \hat{\beta}^{+} \\ q^{+}\end{array}\right]:=\left[\begin{array}{c}\underset{i \in \mathbb{K}}{\operatorname{argmin}} \tilde{x}^{T} P\left(A_{i}(\hat{\beta}) x+a\right) \\ \hat{x}_{2} \\ \hat{\beta} \\ 3-q\end{array}\right] \xi \in \mathcal{D} .}\end{array}\right.$

Note that the fast variables directly impact the stability of the slow variables. However, the jumps do not affect the fast variables, because they do not present any jump.

In order to make a singular perturbation analysis, we will check the assumptions given in [?].

\section{B. Regularity of system's data}

Regularity of system's data comes directly from Proposition1.

\section{Regularity of "manifold"}

The "manifold", which corresponds to the quasisteady-state equilibrium manifold of classical singular perturbation theory [15], that means when $\nu \rightarrow 0^{+}$is

$$
\begin{aligned}
& x_{2}-\hat{x}_{2}=0 \\
& \beta-\hat{\beta}=0 .
\end{aligned}
$$

Note that $\beta-\hat{\beta}=0$ comes from (7). As (21) is continuous, we can take that the manifold is empty outside of $\mathcal{C}$, letting take the following set-valued:

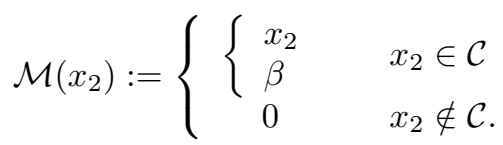

Regard that $\mathcal{M}$ is outer semi-continuous, locally bounded and nonempty.

\section{Stability for reduced system}

The reduced system is the system (10)-(11) in the manifold $\mathcal{M}$, which is

$$
\begin{aligned}
& {\left[\begin{array}{l}
\dot{x} \\
\dot{u} \\
\dot{q}
\end{array}\right]:=\left[\begin{array}{c}
A_{u}(\beta) x+a \\
0 \\
0
\end{array}\right] \xi_{r} \in \mathcal{C}(\mathcal{M})} \\
& {\left[\begin{array}{c}
x^{+} \\
u^{+} \\
q^{+}
\end{array}\right]:=\left[\begin{array}{c}
x \\
\underset{i \in \mathbb{K}}{\operatorname{argmin}} \tilde{x}^{T} P\left(A_{i}(\hat{\beta}) x+a\right) \\
3-q
\end{array}\right] \quad \xi_{r} \in \mathcal{D}(\mathcal{M}) .}
\end{aligned}
$$


being $\xi_{r}=(x, u, q)$. Note there is not jump in $\mathcal{M}$, therefore the reduced system ignores $\hat{x}_{2}$ and $\hat{\beta}$ when determining jumps. Moreover, remark that $q$ nor presents any effect in the reduced system dynamic and neither generates any extra jump. Then, we can guarantee UGAS from [4, Theorem 1].

\section{E. Stability of the boundary layer}

The boundary layer, for each $r$, is given by

$$
\mathcal{H}_{b l}:=\left\{\begin{array}{l}
\dot{\xi}_{1}=0 \\
\dot{\hat{x}}_{2}=\bar{\alpha}\left(u x_{1}-\hat{\beta} x_{2}\right)+\left(x_{2}-\hat{x}_{2}\right) \quad \zeta \in \mathcal{C} \cap r \mathbb{B} \\
\dot{\hat{\beta}}=-\bar{\gamma} x_{2}\left(x_{2}-\hat{x}_{2}\right)
\end{array}\right.
$$

being $r \mathbb{B}$ a closed ball of radius $r$. Note that the boundary layer system ignores the jumps, and during, flows $\xi_{1}$ remains constant.

In order to evaluate the stability of the boundary layer, let consider the error equations of $\mathcal{H}_{b l}$ and re-scale time $t$ to $\tau=\left(t-t_{0}\right) / \nu$, getting

$$
\begin{aligned}
\frac{d}{d \tau} \breve{x}_{2} & =-\bar{\alpha} x_{2 b l} \tilde{\beta}-\breve{x}_{2} \\
\frac{d}{d \tau} \tilde{\beta} & =\bar{\gamma} x_{2 b l} \breve{x}_{2} .
\end{aligned}
$$

which can be rewritten as:

$$
\frac{d}{d \tau} z=J z
$$

with

$$
J=\left(\begin{array}{cc}
-1 & -\bar{\alpha} x_{2 b l} \\
\bar{\gamma} x_{2 b l} & 0
\end{array}\right)
$$

Without lost of generality and from Assumption 1, $x_{2 b l} \in$ $\{\mathbb{R} \backslash\{0\}\}$. Therefore, we can define the next property:

Property 2: The real part of the eigenvalues of $J$, for $x_{2 b l} \in\{\mathbb{R} \backslash\{0\}\}$ are all strictly negative, i.e.

$$
\begin{aligned}
& \lambda_{1}=\mathfrak{R e}\left\{\frac{-1+\sqrt{1-4 \bar{\alpha}^{2} \gamma x_{2}^{2}}}{2}\right\}<0 \\
& \lambda_{2}=\mathfrak{R e}\left\{\frac{-1-\sqrt{1-4 \bar{\alpha}^{2} \gamma x_{2}^{2}}}{2}\right\}<0
\end{aligned}
$$

Proof of theorem 1: From the analysis given in V-A, V-B, V-C, V-D and V-E, we prove ULAS of attractor (19) by applying [?, Theorem 1].

Remark 4: In order to ensure a singular perturbation form, we need to ensure that the observer time response must be larger than the time response of $x$, i.e, $\left|\lambda_{s}\right|<<$ $\alpha$, where $\lambda_{s}$ is the minimum eigenvalue of the slow subsystem.

Remark 5: Note that the real part of the eigenvalues are strictly negative for all $\gamma>0$. However, we deduce that

- the response of the fast variables $\xi_{2}$ is non-oscillating for all

$$
0<\gamma \leq \frac{\bar{\alpha}^{2}}{4 x_{2}^{2} b l}
$$

- likewise, we get oscillations in the transient response of the fast subsystem for

$$
\gamma>\frac{\bar{\alpha}^{2}}{4 x_{2}^{2} b l}
$$

\section{Simulations On Boost CONVERTER}

In this section, we validate our hybrid approach for the boost converter (1) in simulation. These simulations are performed in MATLAB/Simulink by exploiting the HyEQ Toolbox [16].

Consider $V_{i n}=100 \mathrm{~V}, R=2 \Omega, L=500 \mu \mathrm{H}, C_{0}=$ $470 \mu F$,

$$
R_{0}=50 \Omega \in[25,75] \Omega \Rightarrow \beta=0.02 \in[0.0133,004],
$$

which corresponds to $50 \%$ of variation with respect to the nominal value of $R_{0}$ and a sampling time $T_{s}=10^{-6} \mathrm{~s}$.

The switched system state space model (1) is defined by the following matrices:

$$
\begin{gathered}
A_{1}=\left[\begin{array}{cc}
-R / L & 0 \\
0 & -1 / R_{0} C_{0}
\end{array}\right], \quad A_{2}=\left[\begin{array}{cc}
-R / L & -1 / L \\
1 / C_{0} & -1 / R_{0} C_{0}
\end{array}\right], \\
B_{1}=B_{2}=\left[\begin{array}{c}
1 / L \\
0
\end{array}\right] .
\end{gathered}
$$

The set of all attainable equilibrium points are given following [5]

$$
\begin{array}{r}
x_{e}=\left\{\left(i_{e}, v_{e}\right): V_{i n} /\left(R+R_{0}\right) \leq i_{e} \leq V_{i n} / R,\right. \\
\left.v_{e}^{2}+\left(R R_{0}\right) i_{e}^{2}-\left(R_{0} V_{i n}\right) i_{e}=0\right\} .
\end{array}
$$

For our simulations, the chosen equilibrium is

$$
x_{e}=\left[\begin{array}{ll}
i_{e}(\beta) & 120
\end{array}\right]^{T} \text {. }
$$

Following the specification given at the end of Section IV and considering the variation of $\beta$ (23), the cost function is

$J=\min _{u} \sum_{k \in \operatorname{dom}_{j}(\xi)} \int_{t_{k}}^{t_{k+1}} \frac{\rho}{R_{0}}\left(v_{c}(\tau, k)-v_{e}\right)^{2}+R\left(i_{L}(\tau, k)-i_{e}(\beta)\right)^{2} d \tau$

with $\rho=1000$, and

$$
P=\left[\begin{array}{cc}
23.14 & 1.08 \\
1.08 & 37.04
\end{array}\right] \cdot 10^{-2}, Q=\left[\begin{array}{cc}
R & 0 \\
0 & \frac{\rho}{R_{0}}
\end{array}\right]
$$

satisfies Property 1.

Moreover, we take $\eta=0.1$ which corresponds to a sub-optimal value that guarantees a trade-off between performance level and switching frequency, as shown in $[4]$.

Likewise, we select the convergence speed of the observer state, $\alpha$, according to Remark 4 and, having $\left|\lambda_{s}\right|=$ 4000 for the slow sub-system minimum eigenvalue. Then, we need to satisfy $4000<<\alpha$; for this issue, we chose $\alpha=40000$.

Next step is to select the adaptation speed, $\gamma$, according to Remark 5. In a first time, we choose $\gamma$ such that, there is not oscillation during the steady-state $\left(x_{2_{b l}}=\right.$ $\left.x_{e 2}=120\right)$, i.e, $0<\gamma \leq \frac{\bar{\alpha}^{2}}{4 x_{2}^{2}{ }_{b l}}=61 \cdot 10^{-4}$. Then, we take 
$\gamma=5 \cdot 10^{-4}$ in Fig. 3, and $\gamma=50 \cdot 10^{-4}$ in Fig. 4. Finally, we take $\varepsilon=10^{-3}$. Note that in these simulations, the load changes twice, in the transient time at $t=0.001 \mathrm{~s}$ and in the steady state at $t=0.03 \mathrm{~s}$.
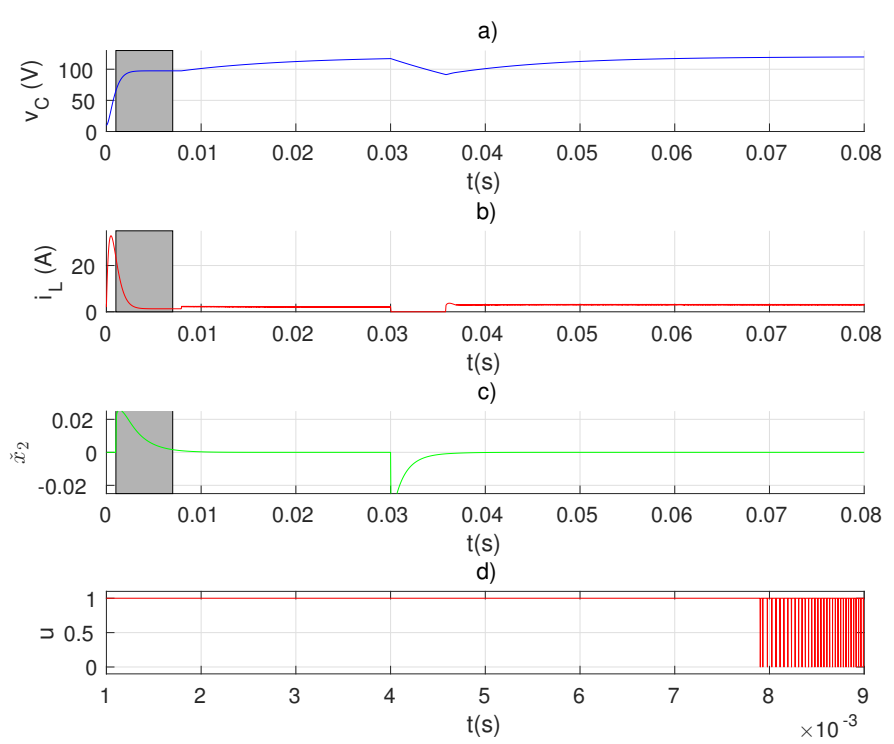

Fig. 3. Evolutions for $\gamma=5 \cdot 10^{-4}$ of the voltage and current in a) and b) resp., $\breve{x}_{2}$ in c) and, zoom of $u$ in d).

a)

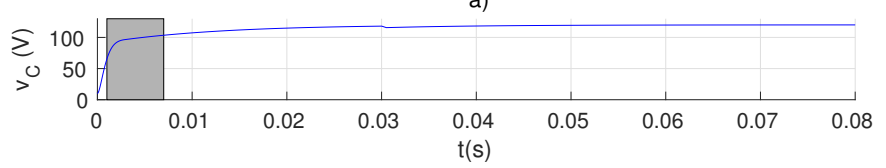

b)

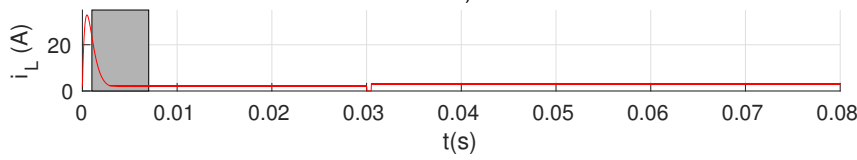

c)
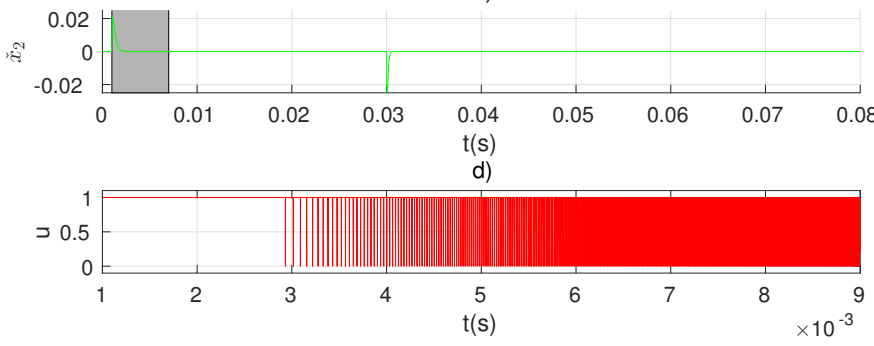

Fig. 4. Evolutions for $\gamma=50 \cdot 10^{-4}$ of the voltage and current in a) and b) resp., $\breve{x}_{2}$ in c) and, zoom of $u$ in d)

Figure 3-a) and 4-a) show the convergence of $x_{2}$ to the equilibrium (24) for any change of $\beta$, which is achieved with $\lambda_{e}=\left[\begin{array}{ll}0.17 & 0.83\end{array}\right]^{T}$ satisfying Assumption 2. Note that during the adaptation of $\beta$, that means, when $\left|\breve{x}_{2}\right| \geq$ $\varepsilon$, at $t=0.001 \mathrm{~s}$ and $t=0.03 \mathrm{~s}$ the states flow with $u$ constant converging to $i_{L, c}, v_{C, c}$, given in (17)-(18). When $\left|\breve{x}_{2}\right| \leq \varepsilon$ the states evolve switching the discrete variable $u$. This evolution can be seen in the zoom of $u$ in Fig. 3-d) and 4-d) for different adaptation speed, $\gamma$. Note that if $\gamma$ is larger the adaptation faster is, according Remark 1.

We can also see during the load changes that, the error $\breve{x}_{2}$ increases, but it converges to zero, due to the fact that $\hat{\beta}$ is adapted to its real value $\beta$, as is established in Lemma 1. Then, we can conclude from Theorem 1 that our attractor (19) is ULAS.

Now, we shows some simulations for the case when the adaptation presents some oscillations in the steady-state, i.e. $\gamma>\frac{\alpha^{2} C_{0}^{2}}{4 x_{2}^{2}}=61 \cdot 10^{-4}$, according to Remark 5. We select $\gamma=0.1$ in Fig. 5 and $\gamma=1$ in Fig. 6. Note that $\breve{x}_{2}$ converges to zero after some oscillations in Fig. 5-c) and in Fig. 6-c), maintaining $x_{2}$ robust w.r.t. its equilibrium value. Wa can also see that the observer convergence is faster, as $\gamma$ larger is.
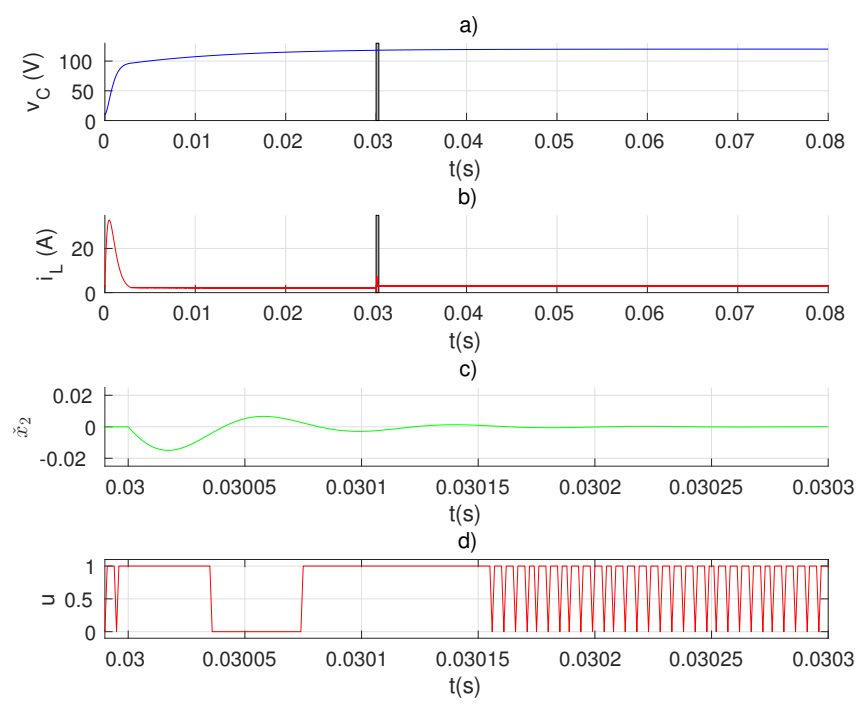

Fig. 5. Evolutions for $\gamma=0.1$ of the voltage and current in a) and b) resp., and zoom of $\breve{x}_{2}$ and $u$ in c) and d) resp.

\section{CONClusions AND FUture WORKS}

A hybrid adaptive control for unknown constant or/and slowly variable load is presented for a boost converter. The method focuses on a hybrid dynamical theory, that considers the real nature of the signals, that means, the continuous-time and the discrete-time signals. On this paradigm an adaptive control is proposed which guarantees the robustness of the voltage in a reference value. This adaptive control is fed by a state observer designed by assuming that the state variables are measured. ULAS of the full system is proven by using a standard singular perturbation analysis and, using Tikhonov's theorem. 
a)
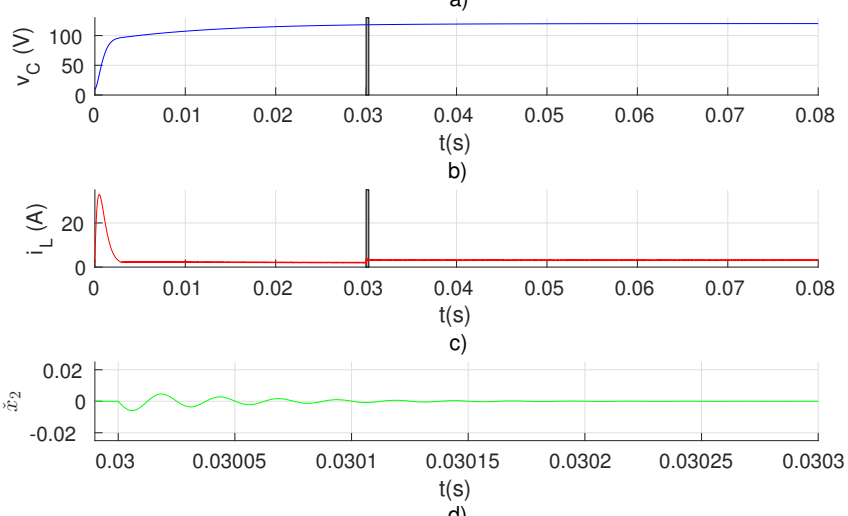

d)

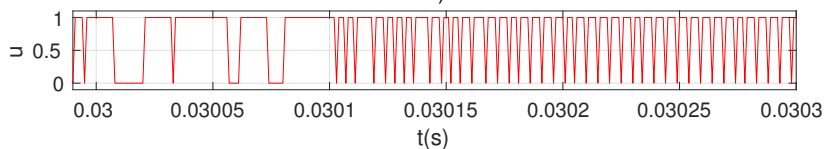

$\mathrm{t}(\mathrm{s})$

Fig. 6. Evolutions for $\gamma=1$ of the voltage and current in a) and b) resp., and zoom of $\breve{x}_{2}$ and $u$ in c) and d) resp.

A future work is to consider a time or space regulation to generate a dwell-time in the controller to reduce the switching in the steady-state.

\section{REFERENCES}

[1] S. S. Muley, R. M. Nargarale . Pwm-based sliding mode control of dc-dc boost converter. International Journal of Advances in Engineering Technology, 6(5):2171-2178, 2013.

[2] P.T. Krein, J. Bentsman, R. M. Bass and B. Lesieutre . On the use of averaging for the analysis of power electronic systems. IEEE Transactions on Industrial Electronics, 60 (8):32913294, 2013.

[3] SC Tan and YM Lai and KT Chi and L. Martínez-Salamero and CK. Wu. A fast-response sliding-mode controller for boost-type converters with a wide range of operating conditions. IEEE Transactions on Industrial Electronics, 54(6):3276-3286, 2007.

[4] C. Albea, G. Garcia and L. Zaccarian. Hybrid dynamic modeling and control of switched affine systems: application to DC-DC converters. In 54th IEEE Conference on Decision and Control, Osaka, Japan, December 2015. An extended version is avaible in: https://hal.archives-ouvertes.fr/hal01220447v3/document.

[5] J.C. Geromel, P. Colaneri and P. Bolzern. Dynamic output feedback control of switched linear systems. IEEE Transactions on Automatic Control,, 2008.

[6] T. AF. Theunisse and J. Chai and R. G. Sanfelice and M. HW. P. Heemels. Robust global stabilization of the dc-dc boost converter via hybrid control. IEEE Transactions on Circuits and Systems I: Regular Papers, 62(4):1052-1061, 2015.

[7] G. J. Jeong, I. H. Kim, Y. I. Son. Application of simple adaptive control to a dc/dc boost converter with load variation. ICCAS-SICE(2009), pages 1747-1751, 209.

[8] H. El Fadil, F. Giri, J.M. Guerrero, B. Salhi. Adaptive control of interleaved boost converter for fuel cell energy. American Control Conference (ACC), 2011, pages 3905-3910, 2011.

[9] H. Sira-Ramirez, R. Tarantino-Alvarado and O. LlanesSantiaco. Adaptive feedback stabilization in pwm- controlled dc-to-dc power sipplies. Internationl Journal of Control, pages 599-625, 1993.

[10] H. El Fadil, F. Giri. Backstepping based control of pwm dc-dc boost power converters. Industrial Electronics, $200 \%$.
ISIE 2007. IEEE International Symposium on, pages 395-400, 2007.

[11] S. Oucheriah and L. Guo . Pwm-based adaptive sliding-mode control for boost dcâĂŞdc converters. IEEE Transactions on Power Electronics, 5(2):182-190, 1990.

[12] C. Albea and F. Gordillo and C. Canudas-de-Wit. Adaptive control design for a boost inverter. Control Engineering Practice, 19(1):32-44, 2011

[13] R. Goebel and R.G. Sanfelice and A.R. Teel. Hybrid Dynamical Systems: modeling, stability, and robustness. Princeton University Press, 2012.

[14] C. Albea, O. Santos, D. Zambrano Prada, F. Gordillo, G. Garcia. Hybrid control scheme for a half-bridge inverter. working paper or preprint, November 2016.

[15] H. K. Khalil. Nonlinear Systems. Prentice Hall, third edition edition, 2002.

[16] R. G. Sanfelice, D. Copp and P. A. Nanez. A toolbox for simulation of hybrid systems in Matlab/Simulink: Hybrid equations (HyEQ) toolbox. In Hybrid Systems: Computation and Control Conference, 2013. 